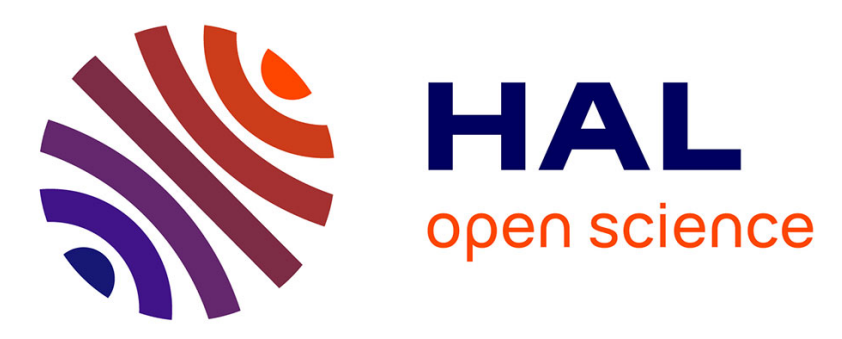

\title{
Analysis of an adaptive strategy for equitably approaching and joining human interactions
}

Vishnu Karakkat Narayanan, Anne Spalanzani, Ren C. Luo, Marie Babel

\section{To cite this version:}

Vishnu Karakkat Narayanan, Anne Spalanzani, Ren C. Luo, Marie Babel. Analysis of an adaptive strategy for equitably approaching and joining human interactions. IEEE Int. Symp. on Robot and Human Interactive Communication, RO-MAN, Aug 2016, New-York, United States. hal-01330889

\section{HAL Id: hal-01330889 \\ https://hal.inria.fr/hal-01330889}

Submitted on 13 Jun 2016

HAL is a multi-disciplinary open access archive for the deposit and dissemination of scientific research documents, whether they are published or not. The documents may come from teaching and research institutions in France or abroad, or from public or private research centers.
L'archive ouverte pluridisciplinaire HAL, est destinée au dépôt et à la diffusion de documents scientifiques de niveau recherche, publiés ou non, émanant des établissements d'enseignement et de recherche français ou étrangers, des laboratoires publics ou privés. 


\title{
Analysis of an adaptive strategy for equitably approaching and joining human interactions
}

\author{
Vishnu K.Narayanan ${ }^{1}$, Anne Spalanzani ${ }^{2}$, Ren C. Luo ${ }^{3}$ and Marie Babel ${ }^{4}$
}

\begin{abstract}
Since social, assistive and companion robots need to navigate within human crowds, understanding spatial social conventions while designing navigation solutions for such robots is an essential issue. This work presents an analysis of an socially compliant robot motion strategy that could be employed by social robots such as humanoids, service robots or intelligent wheelchairs, for approaching and joining humans groups in interaction, and then become an equitable part of the interaction. Following our previous work that formalized the motion strategy, a detailed synthesis is presented here with experiments that validate the proposed system in the real world.
\end{abstract}

\section{INTRODUCTION AND MOTIVATION}

Social robots, particularly in the form of service and companion robots expose a wide range of opportunities for research in mobile robot navigation [1]-[5]. Since it is evident that such robots need to navigate, operate and share physical space between humans, it is essential to assign navigation behaviours in accordance with social expectations.

Considering physical social space management, humans naturally tend to hold social robots with similar spatial social conventions as themselves [6]. Since, detecting, approaching and joining an interacting group of humans remains a fundamental task for a social robot, much emphasis should also be given to the idea of performing this action in an equitable and socially conventional manner. Equitably here refers to the idea of robots being held to the same social standards as humans.

Anthropologist P.E. Hall [7] studied the general conventions and rules followed by humans with respect to physical space management in public and private. He proposed a general proxemic theory describing the spatial distances that individuals maintain in social as well as interpersonal situations. Moreover, he added that physical spatial management by a single person (termed personal space) is different to physical spatial management by a group of persons (termed interaction space). The idea of interaction spaces is then crucial in tackling the problem of a social robot approaching and joining a group of humans as it directly relates to the acceptability of the solution [5] [6].

In [8] we introduced, a feature-based motion strategy that utilized easily extractable features from a detected human group in order to generate a socially acceptable motion to

\footnotetext{
${ }^{1}$ Vishnu K.Narayanan is with Inria Rennes - Bretagne Atlantique, France. vishnu.karakkat-narayanan at inria.fr

2 Anne Spalanzani is with Univ. Grenoble Alpes and Inria Grenoble Rhone Alpes, France

${ }^{3}$ Ren C. Luo is with the National Taiwan University, Taiwan.

${ }^{4}$ Marie Babel is with INSA Rennes and Inria Rennes - Bretagne Atlantique, France

This work is supported by the Inria joint project SAMPEN in collaboration with National Taiwan University.
}

approach and join the group in an equitable manner and to maintain formation. The applicability of the strategy is wide, from humanoid robots to service robots to intelligent wheelchairs. Following our previous work, we here present a detailed analysis focused on the stability, convergence and robustness with a variety of simulated cases. Furthermore, we couple the motion strategy with reactive obstacle avoidance in order to demonstrate its adaptability. Experimental evidence is demonstrated using a mobile robot and the applicability of the approach is discussed with respect to its deployment in real world cases.

\section{COMPARISON TO STATE OF THE ART}

Initial studies using Hall's proxemic theory [7] in social robotics focused on how a robot could safely navigate around social spaces, in some cases to reduce disturbance [9] [1] [2] or in order to make room for human passing [10]. Complementary works began to emerge that investigated how a robot should approach a single human target [11] [12] [13] [3] [4] [5] [14] especially for performing a task such as object handovers [11] [3] or for fetch and carry [5]. These works analysed approach distances, gaze directions, the greeting process etc., for planning socially compliant paths. Within the context of approaching a human/group with the intention of initiating conversation, a fundamental work by Satake et.al. [13], provided a solution in the form of a probabilistic path planning approach that also took into account the predicted trajectory of human motion. In the specific case of approaching a group of people, Althaus et.al. [15] introduced a control scheme that uses the relative distance and orientation of the humans in order to approach and then maintain a formation. Both the works were based on a highly context specific velocity controllers. Thus there exists a need for an adaptive system capable of being deployed without a strong a-priori.

With respect to approaching a group, Karreman et.al [14] conducted a preliminary user study that concluded that executing a frontal approach is more desirable for a group of people in interaction. In this context recent works by Escobedo et. al. [16] [17] have defined an algorithm which is able to calculate meeting points for detected interacting groups. The meeting point was reached by planning a trajectory using an $A^{*}$ algorithm and a Dynamic Window path planner. But traditional robotic objectives such as taking the shortest path to a goal is not always socially conventional and developing planning heuristics based on the proxemic theory state of the art may be tedious [21]. Moreover, in order to integrate other behaviours (possibly high-level) such as local obstacle avoidance, a sensor-based control law offers an 
easy approach. Therefore, the present work analyses a robust feature-based (while sensor-agnostic) control system that is capable of reaching the optimal meeting point in a socially conventional manner. Also, since the control law is featurebased, it is reactive (adaptive) to feature variations in a way planning algorithms may not be.

\section{REVIEW OF THE Motion Strategy}

\section{A. Understanding Interaction Spaces}

A social robot must respect the physical space created when two or more people join to form a focused interaction. When a standing group of humans sustains a single focus of visual and cognitive attention, the resulting interaction is termed as a focused one and the space created is termed as an Interaction Space [18]. Conversations are then said to be focused interactions. The interaction space is comprised of an $\mathrm{O}$-space and a p-space. The $\mathrm{O}$-space is the shared area reserved for the activity that is established by the group: only participants have access to it, they protect it and others tend to respect it [19]. The p-space is the space surrounding the $\mathrm{O}$ space that is used for the placement of the participant bodies and also personal belongings [19]. According to [20], the term F-formation is used to designate the spatial-orientation arrangement and postural behaviours that people create and maintain in order to sustain their O-space. The shape of the Fformation strongly depends on the number of people involved, the relationship among them, the group attentional focus and on environmental constraints. Considering two people in conversation (the most frequent interaction), six formations are common: N-shape, vis-a-vis, V-shape, L-shape, C-shape and side-by-side [19] [20]. Illustrations for vis-a-vis and Vshape formations are given in Figs. 1(a) and 1(c) for reference.

1) Approaching an interaction: Since the aim of the robot is to approach and join a focused interaction, three key points can be asserted in the way it should approach a group.

- The robot should approach in a frontal manner [13].

- The robot should approach without invading the O-space of the interaction.

- A robot should reveal its intention of imminent approach to the group members.

2) Joining an interaction: A geometric representation of the interaction space (i.e the O-space and p-space) for two people formations can be extracted from the position and orientation of the humans (i.e. their F-formation). For two humans $H_{1}$ and $H_{2}$, the geometry of the O-spaces and the p-spaces are given in Figs. 1(b) and 1(d) for vis-a-vis and Vshape formations. $H_{12}$ is the center of the line that joins the two humans, $\phi_{1}$ and $\phi_{2}$ the orientation angles with respect to the arbitrary ground plane frame, $C$ is the center of the $\mathrm{O}$ space and $V_{i}$ is the focus of attention. The distance between the two humans is denoted by $D_{H}$. Judging the point where a robot should place itself in order to become a part of this group is straightforward. Ideally, the robot should position itself on a specific point, within the p-space where the line that joins $H_{12}$ and $V_{i}$ passes through. The robot should also be facing the focus point of the interaction, $V_{i}$. This is the case for all

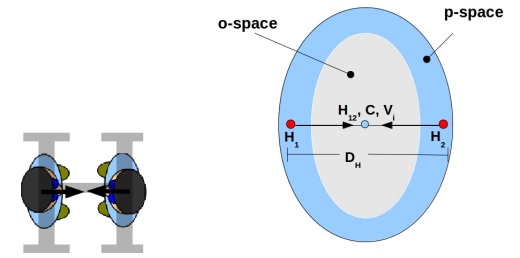

(a) Illustration

(b) Geometric representation

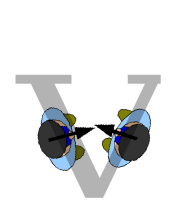

(c) Illustration

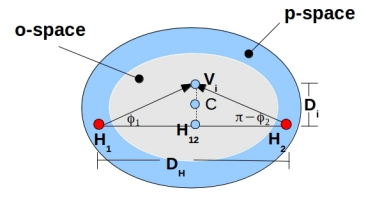

(d) Geometric representation
Fig. 1: A vis-a-vis formation $(\mathrm{a}, \mathrm{b})$ and a $\mathrm{V}$-shape formation $(\mathrm{c}, \mathrm{d})$.

two people formations [9] [21]. Considering formations with more than 2 people, the $\mathrm{O}$-space can be represented as a circle with the focus of attention located at the center of the circle. With regards to calculating the meeting point, a variety of solutions depending on the number, positions and orientations of the participating humans exist. One solution would be to calculate the meeting point by considering the group as a 2 people formation with the 2 people who are farthest in terms of Euclidean distance. A detailed explanation on the representation of interaction spaces as well as on meeting point calculation is given in [9] and [21].

\section{B. Velocity Control Law}

1) Modelling: Assuming a sensor on the robot that perceives the position and orientation of the humans in a specific detected interaction, a fully feature-based control law can be proposed in order to approach and join the specific interaction in a equitable manner.

We model the robot as a non-holonomic unicycle-type robot, which holds for a majority of wheeled service robots such as robotic wheelchairs, while unicycle-type dynamics can be converted to walking motions for humanoids easily as demonstrated in [22]. Thus the control velocity $\mathbf{v}=[u, \omega]^{T}$ comprises of the translational component $u$ and the angular component $\omega$. Assume a rigidly mounted sensor localized within the frame $\mathcal{F}_{s}\left(P_{S}, x_{s}, y_{s}, z_{s}\right)$ (see Fig. 2) perceives and detects interactions in the scene. If the robot frame is denoted by $\mathcal{F}_{r}\left(P_{R}, x_{r}, y_{r}, z_{r}\right)$, then we have a translation vector ${ }^{s} \mathbf{t}_{r}$ and a rotation matrix ${ }^{s} \mathbf{R}_{r}$ between $\mathcal{F}_{s}$ and $\mathcal{F}_{r}$. The relationship between the velocity expressed in the robot frame $\mathbf{v}=[v, 0,0,0,0, \omega]^{T}$ and the velocity expressed in sensor frame $\mathbf{v}_{\mathbf{S}}$ can then be linked via a velocity transformation matrix ${ }^{s} \mathbf{W}_{r}=f\left({ }^{s} \mathbf{R}_{r},{ }^{s} \mathbf{t}_{r}\right)$. Finally the robot Jacobian ${ }^{r} \mathbf{J}_{r}$ expressed in the robot frame can be written as

$$
{ }^{r} \mathbf{J}_{r}=\left[\begin{array}{llllll}
1 & 0 & 0 & 0 & 0 & 0 \\
0 & 0 & 0 & 0 & 0 & 1
\end{array}\right]^{T}
$$


2) Feature Selection: We initially select as features, the $x_{s}$ coordinate $X_{h}$ and the $z_{s}$ coordinate $Z_{h}$, with respect to the sensor frame (see Fig. 2), of the point representing the focus of attention. At the meeting point, $X_{h}$ should attain a desired value of $X_{h}^{*}=0$ which ensures that the sensor is aligned towards the group focus point $V_{i}$. Evidently $Z_{h}$ should reach a desired value $Z_{h}^{*}$ that depends on the type of F-formation in a two people group or on the size of the O-space in a formation with more than two people. But the regulation of the features to their desired value alone does not ensure that the robot reaches the optimal meeting point (since the final meeting point can lie on any point on the circle with radius $Z_{h}^{*}$ around the group), nor can it ensure that it takes a socially acceptable trajectory.

Therefore we introduce a third feature, $L_{h}$, termed as the group length. In two-people formations, $L_{h}$ is the projection, on $x_{s}$, of the line segment connecting the two humans $H_{1}$ and $H_{2}$. In groups with more that two people $L_{h}$ is the projection, on $x_{s}$, of the line segment connecting the two farthest humans (in terms of Euclidean distance). The feature $L_{h}$ should reach a value of $L_{h}^{*}=D_{H}$ in two-people formations and $D_{O}$ (the diameter of the $\mathrm{O}$-space) in groups with more that two people. It can be seen that at only at the meeting point, the three features are at their desired values. Moreover all three features can be easily extracted once the robot is able to perceive humans.

3) Control law: To design a control strategy for socially compliant trajectory generation, we can define two tasks namely $\mathbf{e}_{\mathbf{1}}=\left[Z_{h}-Z_{h}^{*}, X_{h}-X_{h}^{*}\right]^{T}$ and $\mathbf{e}_{\mathbf{2}}=\left[L_{h}-L_{h}^{*}\right]$. If $\mathbf{L}=\left[\mathbf{L}_{Z_{h}}, \mathbf{L}_{X_{h}}, \mathbf{L}_{L_{h}}\right]^{T}$ represents the interaction matrix that relates the dynamics of the features $Z_{h}, X_{h}$ and $L_{h}$ with respect to the sensor velocity screw $\mathbf{v}_{\mathbf{s}}$, the Jacobians $\mathbf{J}_{1}$ and $\mathbf{J}_{2}$ respectively related to $\mathbf{e}_{\mathbf{1}}$ and $\mathbf{e}_{\mathbf{2}}$ can be expressed as:

$$
\begin{aligned}
& \mathbf{J}_{1}=\left[\begin{array}{c}
\mathbf{J}_{Z_{h}} \\
\mathbf{J}_{X_{h}}
\end{array}\right]=\left[\begin{array}{lll}
\mathbf{L}_{X_{h}}{ }^{s} \mathbf{W}_{r}{ }^{r} \mathbf{J}_{r} \\
\mathbf{L}_{Z_{h}}{ }^{s} \mathbf{W}_{r}{ }^{r} \mathbf{J}_{r}
\end{array}\right] \\
& \mathbf{J}_{2}=\mathbf{J}_{L_{h}}=\left[\mathbf{L}_{L_{h}}{ }^{s} \mathbf{W}_{r}{ }^{r} \mathbf{J}_{r}\right] .
\end{aligned}
$$

The aim is then to realize the two tasks simultaneously (i.e. minimize task $1\left(\mathbf{e}_{\mathbf{1}}\right)$ and task $2\left(\mathbf{e}_{2}\right)$ to zero) while ensuring spatial social constraints. But we can see that each task $\left(\mathbf{e}_{1}\right.$ and $\left.\mathbf{e}_{2}\right)$ constrains both the DOFs of the robot (as shown in [8]). Therefore realising an optimal switching from task $\mathbf{e}_{\mathbf{1}}$ to task $\mathbf{e}_{\mathbf{2}}$ at specific intervals can ensure their simultaneous convergence, as well as ensure a constrained socially complaint trajectory generation. This is achieved by using a continuous diagonal matrix $\mathbf{H}=\operatorname{Diag}\left(h_{Z_{h}}, h_{X_{h}}\right)$ that weights the task $\mathbf{e}_{1}$, and where $h_{Z_{h}}$ and $h_{X_{h}} \in[0 ; 1]$ are the varying weights respectively associated to the features $Z_{h}$ and $X_{h}$. This allows the activation of task $\mathbf{e}_{\mathbf{1}}$ at only specific intervals. Consequently task $\mathbf{e}_{2}$ can be projected onto the null space of task $\mathbf{e}_{\mathbf{1}}$ using a projection operator $\mathbf{P}_{H}$. This is achieved using the following control strategy proposed in [8]:

$$
\mathbf{v}=-\lambda\left[\mathbf{J}_{1}^{\oplus \mathbf{H}} \mathbf{e}_{\mathbf{1}}-\mathbf{J}_{2}^{\mathbf{P}_{H}}\left(_{\left.\left(\mathbf{e}_{\mathbf{2}}-\mathbf{J}_{2} \mathbf{J}_{1}^{\oplus \mathbf{H}} \mathbf{e}_{\mathbf{1}}\right)\right] .} .\right.\right.
$$

$\mathbf{J}_{1}^{\oplus \mathbf{H}}$ represents the continuous inversion of the weighted matrix product $\mathbf{H} \mathbf{J}_{1}$ and thus the projection operator $\mathbf{P}_{H}=$ $\mathbb{I}_{2}-\mathbf{J}_{1}^{\oplus \mathbf{H}} \mathbf{J}_{1}$. Here $\mathbb{I}_{2}$ represents the Identity matrix. Moreover $\mathbf{J}_{2}^{\mathbf{P}_{H}} \oplus$ represents the continuous inversion of the product $\mathbf{P}_{H} \mathbf{J}_{2}$. The theoretical bases and proof of continuity of these operators are provided in [23]. Finally $\lambda$ in (3) denotes the control gain.

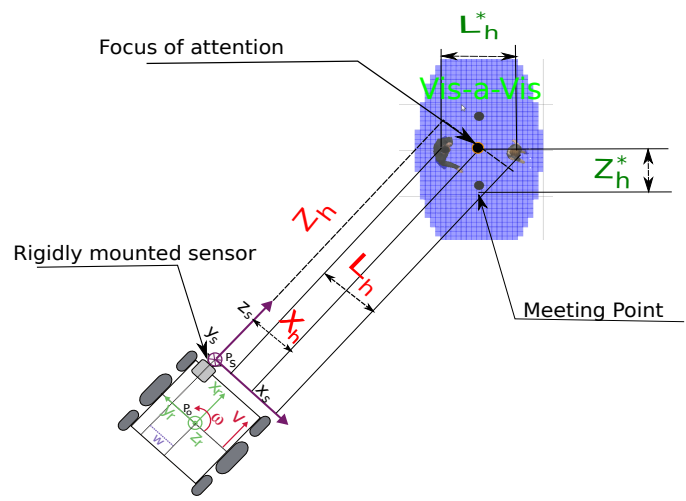

Fig. 2: A unicycle-type robot with respect to vis-a-vis group. Robot and sensor frames along with the geometric representation of the features.

The weighting matrix $\mathbf{H}=\operatorname{Diag}\left(h_{Z_{h}}, h_{X_{h}}\right)$ takes up the following form presented in Figures 3 and 4 . The interval

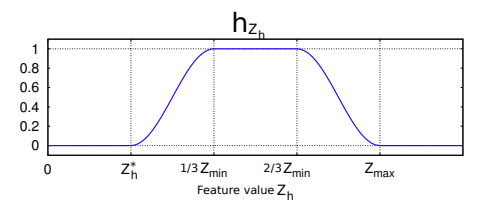

Fig. 3: The evolution of $h_{Z_{h}}$ with respect to the feature $Z_{h}$

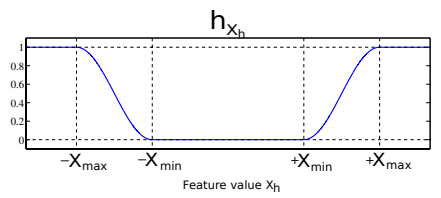

Fig. 4: The evolution of $h_{X_{h}}$ with respect to the feature $X_{h}$

parameters for $h_{Z_{h}}$ are $Z_{\max }=\sqrt{X_{\text {init }}^{2}+Z_{\text {init }}^{2}}$ and $Z_{\text {min }}=$ $Z_{\max }-Z_{h}^{*}$ with $X_{\text {init }}$ and $Z_{\text {init }}$ being the estimations of $X_{h}$ and $Z_{h}$ at the first instant when $Z_{h}>0$. For $h_{X_{h}}$, $X_{\text {min }}=k_{1}\left|\mathbf{e}_{2}\right|$ and $X_{\max }=k_{2} X_{\min }$ represent the interval parameters with $k_{1}$ and $k_{2}$ being two constants tuned according to the field of view (FOV) of the sensor ${ }^{1}$.

\section{ANALYSIS}

We analyse the control strategy presented above with respect to its stability, convergence and robustness. The scenario is designed as a robotic wheelchair approaching a two-person interaction for a variety of configurations. All the simulations presented are modelled within the ROS middleware.

\footnotetext{
${ }^{1} k_{1}$ and $k_{2}$ were empirically set according to the formula $k_{1}=$ fov $/ 120$ and $k_{2}=1 \frac{1}{3} k_{1}$. Where fov is the field of view of the sensor in degrees. It can be postulated that this assignment is valid for any sensor upto $180^{\circ}$ field of view.
} 


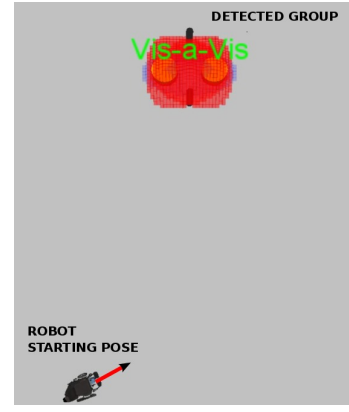

(a) Case I - Start

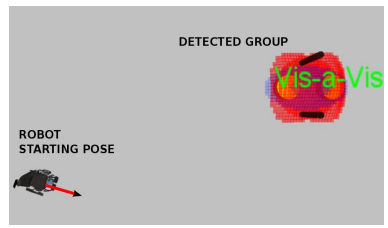

(c) Case II - Start

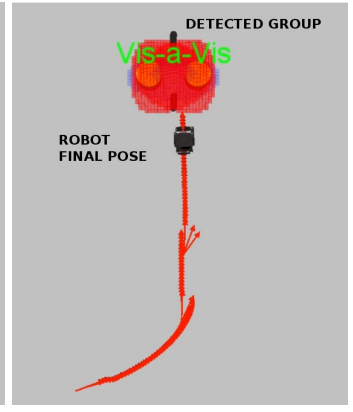

(b) Case I - End

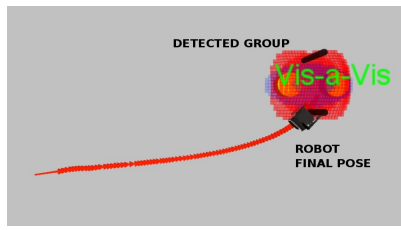

(d) Case II - End
Fig. 5: The starting and final robot poses with respect to a detected group for two simulation cases.

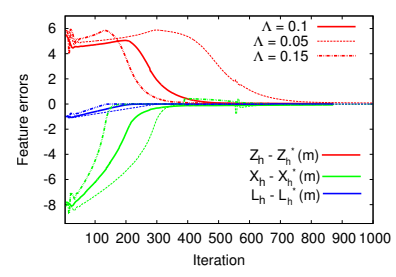

(a) Case I

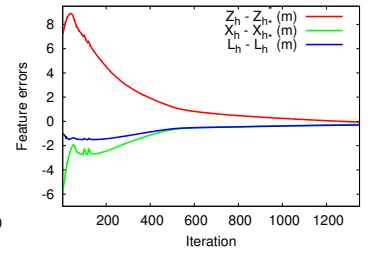

(b) Case II
Fig. 6: The evolution of the feature errors. (a) 3 runs of Case I with different gains and, (b) Case II with gain $\lambda=0.1$.

\section{A. Stability}

From [23], stability of the control law (3) can be asserted for two conditions viz: asymptotic stability in the sense of Lyapunov at binary activation (i.e $\forall i=X_{h}, Z_{h}, h_{i}=0$ or $h_{i}=1$.) and local asymptotic stability around the desired position. These assertions are true if the tasks $\dot{e}_{\mathbf{1}}=\mathbf{J}_{1} \mathbf{v}$ and $\dot{\mathbf{e}_{2}}=\mathbf{J}_{2} \mathbf{v}$ are locally asymptotically stable. Let $V=\frac{1}{2} \mathbf{e}_{\mathbf{i}}{ }^{T} \mathbf{e}_{\mathbf{i}}$ $\forall i=1,2$, be a positive continuous Lyapunov candidate, then the task $\mathbf{e}_{\mathbf{i}}$ is said to be asymptotically stable if $\dot{V}<0$. Now, $\dot{V}=-\mathbf{e}_{\mathbf{i}}^{T} \mathbf{J}_{i} \mathbf{J}_{i}^{+} \mathbf{e}_{\mathbf{i}}$. Therefore if $\mathbf{J}_{i} \mathbf{J}_{i}^{+}$is positive definite, then $\dot{V}<0$. This condition is true (since the product is Identity) as per the definition of the Jacobians $\mathbf{J}_{1}$ and $\mathbf{J}_{2}$ (see [8]) and as the interaction matrix $\mathbf{L}$ is well defined. The only singularity occurs when the robot starts at a position in the same hyperplane as, and directly facing, the interacting humans (i.e. $L_{h}=0$ ). This case can be resolved by moving the robot in open loop till an non-singular position is reached.

\section{B. Convergence}

In order to analyse the convergence, the robot is tasked with joining the group at a variety of random initial positions. The control gain $\lambda$ was set at 0.1 unless otherwise specified. Table
TABLE I: Absolute error values at the final position - 12 random initial positions

\begin{tabular}{c||c|c|c|c}
\hline Error & Mean (m) & Low (m) & High (m) & Std. Dev (m) \\
\hline \hline$\left|Z_{h}-Z_{h}^{*}\right|$ & 0.091 & 0.012 & 0.233 & 0.081 \\
$\left|X_{h}-X_{h}^{*}\right|$ & 0.066 & 0.012 & 0.230 & 0.065 \\
$\left|L_{h}-L_{h}^{*}\right|$ & 0.089 & 0.002 & 0.251 & 0.092 \\
\hline
\end{tabular}

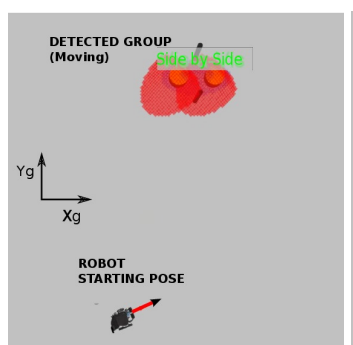

(a) Start

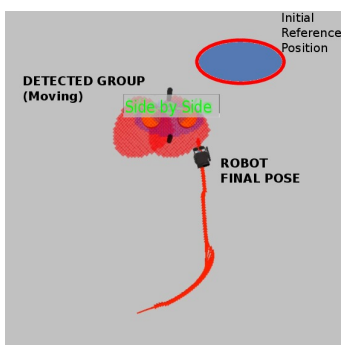

(b) End
Fig. 7: The starting and final robot poses with respect to a continuously moving detected group.

I provides the statistics for the feature errors (i.e $Z_{h}-Z_{h}^{*}$, $X_{h}-X_{h}^{*}$ and $L_{h}-L_{h}^{*}$ ) at the final robot pose (i.e at the meeting point) for 12 different trials. It can be seen that the mean feature error at the final pose is low thus proving that the control law is able to converge to the desired pose satisfactorily. The maximum feature error was around $24 \mathrm{~cm}$ in some cases due to the robot starting at an almost singular position as represented in Fig. 5c. Fig. 5 shows two cases of the task where in case I, the robot starts in a straightforward position and in case II, the robot starts in an almost singular position. It can be seen that the meeting point is reached in both the cases. The evolution of the errors in Fig. 6 also corroborate this fact. Moreover, case I is demonstrated in Fig. 6 with three different control gains where a three degrees of magnitude change in $\lambda$ does not affect the convergence.

\section{Robustness}

In order to assess the robustness of the control strategy, two scenarios are presented. In both the scenarios the robot is tasked with joining a two person side-by-side group from a starting configuration as presented in case I (Fig. 5a). The first scenario involves the robot approaching and joining a group which is moving at a constant speed of $0.1 \mathrm{~m} / \mathrm{s}$ at an angle of $-135^{\circ}$ with respect to a $2-\mathrm{D}$ reference global frame (see Fig. 7). In such a case, even though the group is in continuous motion, the robot is able to reach a satisfactory position (Fig. 7b) in order to become an equitable part of the interaction. It has to be noted that the group remains in motion even as the robot closes in to the meeting point. This is necessarily not the case in the real world. But with respect to the control law adapting to the motion of the group, this scenario provides a clear evidence of the robustness of the proposed solution.

The second scenario involves the robot tasked with joining a group in the presence of dynamic humans. An ultrasoundbased reactive collision avoidance mechanism [25] was employed on top of the proposed motion strategy. The collision 


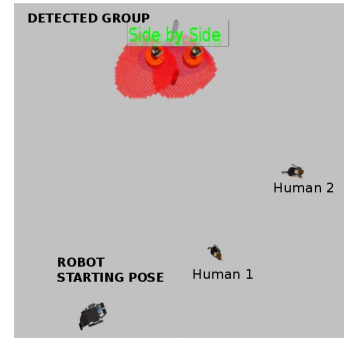

(a) Start

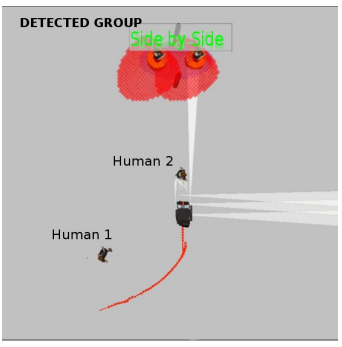

(c) Encounter with Human 2

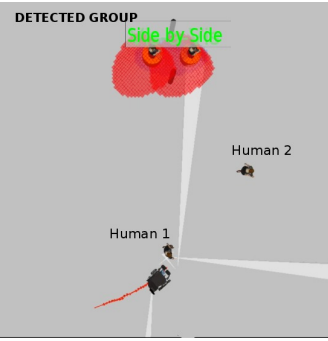

(b) Encounter with Human 1

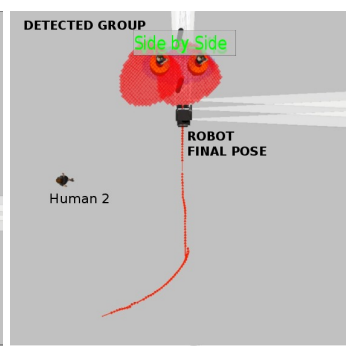

(d) End
Fig. 8: The robot trying to approach and join a group in the presence of other moving humans.

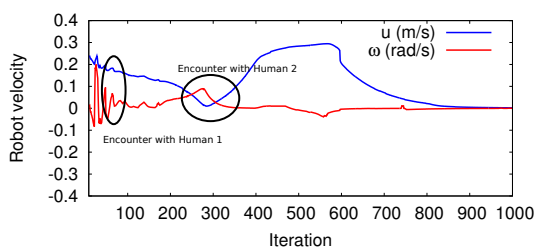

Fig. 9: The evolution of the robot translational $(u)$ and angular $(\omega)$ velocities during the simulation for the above Case.

avoidance mechanism essentially constrains the velocities coming from the velocity controller (3) using information coming from the sensors. The technical details of the final controller is not delineated here as it is not an objective of this work. Figs. 8 demonstrate that the robot is able to avoid collision with the moving humans while attempting to join the group. This is corroborated from the velocity plots (Fig. 9) that show variation during human encounters. During the first encounter, the angular velocity is erratic due to the robot simultaneously turning towards the group while attempting to avoid collision with the human. This is caused by the reactive controller generating opposite velocity commands to the control strategy. It can be essentially be resolved by using a higher level decision making algorithm. But in the second encounter, it can be seen that the robot slows down (see Fig 9) to accommodate the human passing.

\section{Experiments}

Trials using a mobile robot were carried out in order to validate the system in a noisy real world situation. A mobile robot equipped with a laser scanner capable of detecting and classifying humans based on a leg detection algorithm was used for testing. The robot was localised using the said laser and odometry within a map generated online using a widely implemented SLAM scheme [26]. Again the framework was designed within a ROS architecture while the computations were performed using the ViSP software. It was tasked with approaching and joining the two person human interaction at random initial positions. The robot was able to reach the optimal meeting point based on the spatial constraints described in Sec. IIIA by taking an appropriate trajectory towards the interaction (see Fig. 10). As designed, the robot is always gazing at the group during the motion. Particularly in the third run, where the robot is initially gazing away from the group. The specific definition of the weight matrix $\mathbf{H}$ allows the robot to turn towards the group initially and approach in a frontal manner order to join the group. Also, the robot does not encroach the $\mathrm{O}$-space of the interaction thus matching all the constraints presented in Sec. IIIA Part 1.

\section{E. Further Discussion}

1) Adaptability: It can be asserted that the motion strategy analysed here is adaptable and modular in the sense that such a feature based design allows adaptation of the system into a variety of general cases. It is due to the fact that the features presented here are easily extractable once the robot is able to detect people. As such a non-holonomic constraint on the robot applies to the majority of social and service robots. Moreover, the framework developed within a ROS system facilitates redistribution and widespread usage.

2) Context Dependency: With respect to applying the control law within a variety of contexts, for example esoteric cases like a group waiting in line, the theory that is derived from proxemics along with the features presented here can be modified in order to accommodate such contexts. The control strategy (i.e. the features) does not depend on the type of group nor does it take into account the perception of the humans with the group. Thus context specific behaviours can be easily integrated onto the system.

3) Group Perception: Perception of the group is a vital issue to be tackled when designing such algorithms for social robots and thus a motivation for future work. It would be very interesting to assess the human perception of the path chosen by the robot and perform a subjective analysis. This can also help in adapting the algorithm for a variety of context specific behaviours.

\section{Conclusion}

The paper analyses an adaptive control strategy that can be employed by a social mobile robot in order to approach and join an interacting group. The analyses demonstrate modularity, convergence and robustness with respect to a variety of cases and also verify the efficacy of the system in real and dynamic world. A public ROS package for the control strategy will be published soon. Further studies aim at assessing the control strategy with the intention of the group in mind. A real world application is being designed where user input coming from a wheelchair user is blended with the proposed framework in order to create a semi-autonomous assistive wheelchair system for human-aware navigation. 

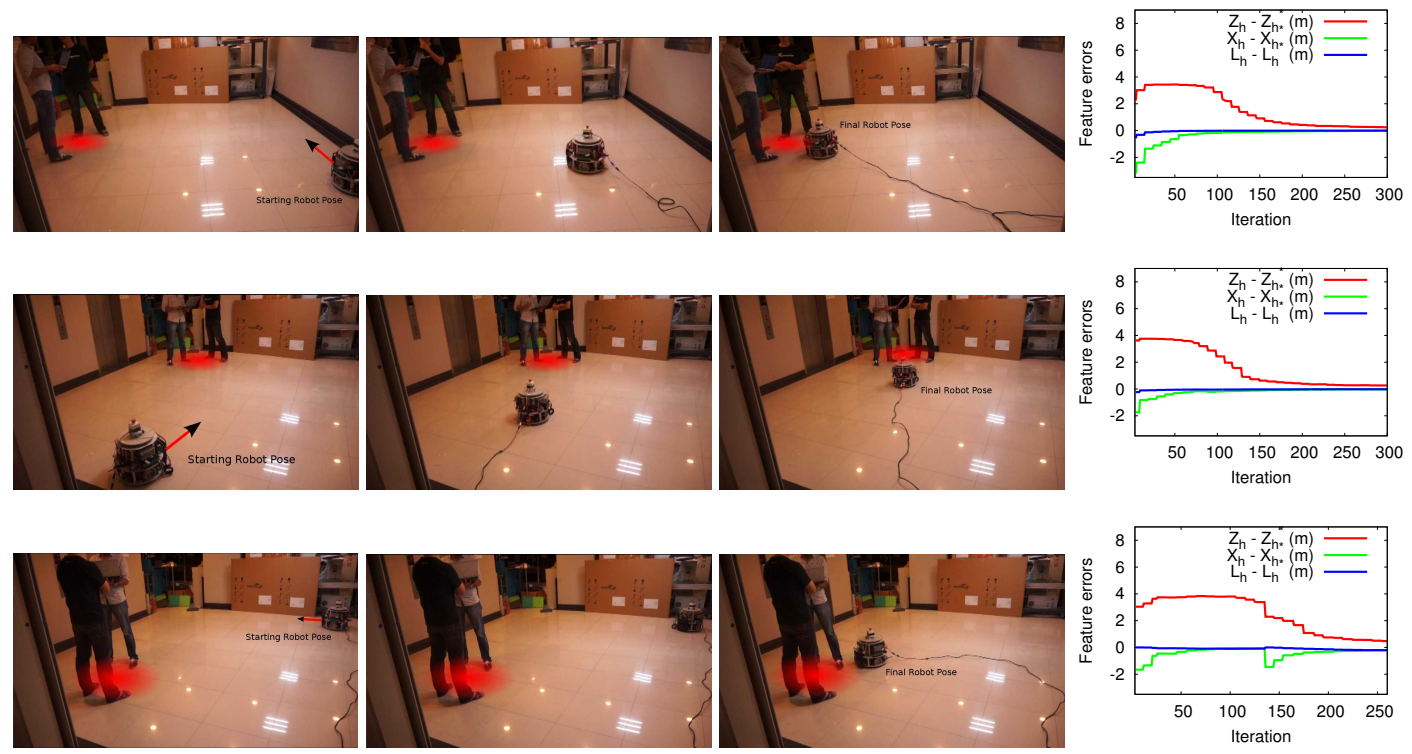

Fig. 10: Snapshots of the starting robot pose, a moment during the motion and the final robot pose for 3 experimental trials (each row). The robot is able to reach the desired position without encroaching the O-space of the interaction. The evolution of the feature errors is also plotted alongside.

\section{REFERENCES}

[1] E. Sisbot, A. Clodic, L. Marin U., M. Fontmarty, L. Brethes, and R. Alami, "Implementing a Human-Aware Robot System," in The 15th IEEE Int. Symp. on Robot and Human Interactive Communication , 2006, pp. 727-732.

[2] E. Sisbot, R. Alami, T. Simeon, K. Dautenhahn, M. Walters, and S. Woods, "Navigation in the presence of humans," in 5th IEEE-RAS Int. Conf. on Humanoid Robots, 2005.

[3] J. Balasuriya, K. Watanabe, and A. Pallegedara, "Giving robots some feelings towards interaction with humans in ubiquitous environment," in Int. Conf. on Industrial and Information Systems, 2007, pp. 529-534.

[4] E. Sisbot, A. Clodic, R. Alami, and M. Ransan, "Supervision and motion planning for a mobile manipulator interacting with humans," in 3rd ACM/IEEE Int. Conf. on Human-Robot Interaction ,2008, pp. 327-334.

[5] M. Walters, K. Dautenhahn, S. Woods, and K. Koay, "Robotic etiquette: Results from user studies involving a fetch and carry task," in 2 nd ACM/IEEE Int. Conf. on Human-Robot Interaction, 2007, pp. 317-324.

[6] C. Breazeal, A. Takanishi, and T. Kobayashi, "ch. Social Robots that Interact with People" Springer Handbook of Robotics. Springer Berlin Heidelberg, 2008, , pp. 1349-1369.

[7] E. T. Hall, "The hidden dimension", Doubleday, 1969.

[8] V. K. Narayanan, A. Spalanzani, F. Pasteau, and M. Babel, "On equitably approaching and joining a group of interacting humans," in IEEE/RSJ Int. Conf. on Intelligent Robots and Systems, 2015, pp. 40714077.

[9] J. Rios-Martinez, A. Spalanzani, and C. Laugier, "Understanding human interaction for probabilistic autonomous navigation using Risk-RRT approach," in IEEE/RSJ Int. Conf. on Intelligent Robots and Systems. 2011, pp. 2014-2019.

[10] E. Pacchierotti, H. Christensen, and P. Jensfelt, "Design of an OfficeGuide Robot for Social Interaction Studies," in IEEE/RSJ Int. Conf. on Intelligent Robots and Systems (IROS). 2006, pp. 4965-4970.

[11] K. Dautenhahn, M. Walters, S. Woods, K. L. Koay, C. L. Nehaniv, A. Sisbot, R. Alami, and T. Siméon, "How may I serve you?" in $1 s t$ ACM SIGCHI/SIGART Conf. on Human-robot interaction. 2006, p. 172.

[12] K. W. Strabala, M. K. Lee, A. D. Dragan, J. L. Forlizzi, S. Srinivasa, M. Cakmak, and V. Micelli, "Towards Seamless Human-Robot Handovers," Journal of Human-Robot Interaction, vol. 2, no. 1, pp. 112-132, 2013.

[13] S. Satake, T. Kanda, D. Glas, M. Imai, H. Ishiguro, and N. Hagita, "How to approach humans?-strategies for social robots to initiate interaction" in ACM/IEEE Int. Conf. on Human-Robot Interaction, 2009 pp. 109116.

[14] D. Karreman, L. Utama, M. Joosse, M. Lohse, B. van Dijk, and V. Evers, "Robot etiquette: How to approach a pair of people?" in Proceedings of the 2014 ACM/IEEE Int. Conf. on Human-robot Interaction, 2014, pp. 196-197.

[15] P. Althaus, H. Ishiguro, T. Kanda, T. Miyashita, and H. Christensen, "Navigation for human-robot interaction tasks," in IEEE Int. Conf. on Robotics and Automation, vol. 2, 2004.

[16] A. Escobedo, A. Spalanzani, and C. Laugier, "Multimodal control of a robotic wheelchair: Using contextual information for usability improvement," in IEEE/RSJ Int. Conf. on Intelligent Robots and Systems. 2013, pp. 4262-4267.

[17] A. Escobedo, A. Spalanzani, and C. Laugier, "Using social cues to estimate possible destinations when driving a robotic wheelchair" in IEEE/RSJ Int. Conf. on Intelligent Robots and Systems. 2014, pp. 3299-3304.

[18] Erving Goffman, Behavior in Public Places: Notes on the Social Organization of Gatherings. Free Press, 1966.

[19] A. Kendon, Development of Multimodal Interfaces: Active Listening and Synchrony, S Springer Berlin Heidelberg, 2010, vol. 5967.

[20] T. M. Ciolek and A. Kendon, "Environment and the Spatial Arrangement of Conversational Encounters," Sociological Inquiry, vol. 50, no. 3-4, pp. 237-271, 1980.

[21] Jorge Rios-Martinez, "Socially-Aware Robot Navigation: combining Risk Assessment and Social Conventions, Ph.D. Thesis, University of Grenoble, 2013.

[22] A. Faragasso, G. Oriolo, A. Paolillo, and M. Vendittelli, "Vision-based corridor navigation for humanoid robots," in IEEE Int. Conf. on Robotics and Automation. 2013, pp. 3190-3195.

[23] N. Mansard, A. Remazeilles, and F. Chaumette, "Continuity of VaryingFeature-Set Control Laws," IEEE Trans. on Automatic Control, vol. 54, no. 11, pp. 2493-2505, 2009.

[24] E. Marchand, F. Spindler, and F. Chaumette, "ViSP for visual servoing: a generic software platform with a wide class of robot control skills," IEEE Robotics and Automation Magazine, vol. 12, no. 4, pp. 40-52, 2005.

[25] M. Babel et. al., "HandiViz project: clinical validation of a driving assistance for electrical wheelchair," in IEEE Workshop On Advanced Robotics And Its Social Impacts, 2015.

[26] G. Grisetti, C. Stachniss, and W. Burgard, "Improved techniques for grid mapping with rao-blackwellized particle filters," IEEE Trans. on Robotics, vol. 23, no. 1, pp. 34-46, 2007. 